\title{
Efficiently Irrational: Illuminating the Riddle of Human Choice
}

Paul W Glimcher*, PhD

Neurosciences Institute

New York University Grossman School of Medicine

New York, NY 10016

\section{Abstract}

For the past half century cognitive and social scientists have struggled with the irrationalities of human choice behavior; people consistently make choices that are logically inconsistent. Is human choice behavior evolutionarily adaptive or is it an inefficient patchwork of competing mechanisms? This review presents an interdisciplinary synthesis arguing for a novel interpretation; choice is efficiently irrational. Connecting findings across disciplines suggests that observed choice behavior reflects a precise optimization of the trade-off between the costs of increasing the choice mechanism's precision and the declining benefits that come as precision increases. Under these constraints a rationally imprecise strategy emerges which works towards optimal efficiency, rather than towards optimal rationality. This approach rationalizes many of the puzzling inconsistencies of human choice behavior, explaining why these inconsistencies arise as an optimizing solution in biological choosers.

*Correspondence: paulg@nyu.edu (Paul Glimcher)

Highlights

Glossary 


\section{The Riddle of Human Choice}

Nicholas William Leeson was a trader at London's Barings' Bank, leading a unit at the Singapore stock exchange. By the end of 1992, Nick's group accounted for $10 \%$ of Barings' annual world-wide profit. That year, however, one of Nick's traders sold when she was instructed to buy, accidentally loosing $£ 20,000$. Facing this administrative loss, Nick began to make secret trades, some risker than usual, to cover his deficit before it was discovered. These riskier-than-usual trades sometimes worked out, but sometimes led to greater losses. As his losses (and presumably his anxiety) grew, Nick became more and more willing to accept risky trades with lower and lower probabilities of success. By late 1993 , Nick had clandestine losses of $£ 23$ million, by 1994 his hidden losses had grown to $£ 208$ million.

To cover these losses, on January 16,1995 , Nick agreed to a particularly risky trade, betting a huge amount on the overnight stability of the Tokyo stock market. At 5:46am that morning, a major earthquake struck Japan, decimating the Tokyo markets and Nick's accounts. Now facing truly massive losses, Nick bet more and more each day that the Nikkei average would suddenly recover. By the morning of February $23^{\text {rd }}$ Nick had lost $£ 827$ million, bankrupting the oldest merchant bank in the United Kingdom (Rawnsley and Leeson, 1994).

Scholars and philosophers (eg., Pascal, 1623:1948; Arnaud and Nicole, 1662:1996) have generally assumed that people are trying to maximize, sometimes money, sometimes love, and sometimes something else, with their choices. Pascal, for example, hypothesized we strive to maximize long-run the accumulation of these articles, we maximizer their expected value. Unfortunately, the observation that humans often sacrifice higher expected value to avoid risk largely disproved this conjecture in the $18^{\text {th }}$ century (Bernoulli, 1738:1954). This led mathematicians to hypothesize that instead, human choice in all its complexity was aimed at maximizing a more subjective notion of accumulation, expected utility. The first half of the $20^{\text {th }}$ century saw a general effort to narrow down the set of all reasonable algorithms for maximization under this assumption by focusing on the notion that well-organized patterns of choice must be transitive: If a human chooser prefers feeling loved to owning cars, and owning cars to pastrami sandwiches, then she simply cannot also prefer pastrami sandwiches to love (Box 1). If she does, then it can be proven that her choices cannot achieve any goal. No stable maximization (or utility) function can ever be used to describe (or justify) intransitive choice (eg., Samuelson, 1937; Houthakker, 1950). This critical insight led economists to refine definitions of the behavioral signatures of goal-directed decisionmaking, focusing on the notion that choices can be meaningful only if they maximize a subjective utility function, a pattern now referred to technically by economists as rational choice (Box 2). 
The extension of this approach from to the domain of risky choice (von Neumann and Morgenstern, 1944; Savage 1954) allows us to show that Nick Leeson's decisions were technically "irrational" in just this sense. It is reasonable for a decision-maker to choose to tolerate or abjure risk. Either preference can lead to coherent, logically consistent, and transitive choice behavior, maximizing a subjective notion of value that is rational in the technical sense. What Nick Leeson did, however, falls outside that category. His decision criteria, regardless of whether they were driven by emotions or logic, were changing dynamically in a destructive feedback loop that induced a riskrelated intransitivity. As his account balances dropped lower and lower below his lawful balance, a key reference point, Nick changed his decision-making process. What he rejected one day as too risky, he preferred on the next day, driven by the widening gap between his balances and his reference point. This fundamental inconsistency, the neoclassical economists showed, meant that Nick's behavior could not be seen as maximizing or goal-directed. No coherent hidden agenda or (continuous monotonic and stable) subjective function was being pursued.

In the last 50 years it has become abundantly clear that this kind of intransitivity, an intransitivity mathematically associated with an internally or externally held reference point, is a key feature of human decision-making (Kahneman and Tversky, 1979; Kahneman et al., 1982; Koszegi and Rabin, 2006; 2007). This is not the only form of inconsistency that is widely observed either. Human decision-makers systematically treat losses relative to the shifting reference point differently than they treat gains, and they distort their representations of outcome probabilities (Allais, 1953; Kahneman and Tversky, 1979; Wu and Gonzalez, 1998).

Until quite recently, the standard explanation for these puzzling failures-tomaximize rested on the notion that human choosers were intransitive because decisionmakers were actually built from a number of independent modules (multiple-selves), some emotional and others coldly logical. While each module (even the emotional ones) might be fully transitive when operating on its own, these multiple-selves competed for control of the decision-maker's actions and created inconsistencies as the mechanisms traded-off control between one another (eg., Tversky and Kahneman, 1986; Laibson, 1997). According to this view, having different and potentially incompatible risk attitudes for losses and gains emerged from the fact that different, and slightly incompatible, neurobiological or psychological modules are assumed to be independently responsible for choices over gains and choices over losses.

Over the past two decades, however, a revisionist interdisciplinary approach has begun to suggest that humans may not be as irrationally intransitive as they at first appeared (Sims, 2003; Glimcher, 2011; Caplin at al 2011; Tajima et al., 2019; Polonia et al. 2019; Steverson et al., 2019; Juechems et al., 2021, Khaw et al, 2021). These data are beginning to suggest that humans, emotions and all, may not only be much 
more goal directed in their maximization than is implied by behavioral economics, they may be much closer to the objective optimizers that Pascal imagined than the neoclassical economists imagined.

Building on Herb Simon's $(1955 ; 1957)$ insight that biological constraints are critical to understanding human choice, this neuroeconomic (Glimcher and Fehr, 2013) approach has suggested that economists may have mis-specified the problem human decision-makers face. The neoclassical economists treated humans as abstract computational machines that could represent (and store) the subjective utilities of every option with infinite precision. They assumed choosers could perfectly weigh these precise representations against one another. They assumed that the human decisionmaking machinery was insensitive to the real biological costs of storing information, representing value, and computing choice.

We know, however, that precision in neural computation is metabolically costly and that increasing the precision of representation, storage and computation necessarily increases costs. This notion, that at an evolutionary timescale the benefits of precision must trade-off against the costs of representational accuracy, suggests that the imprecision in our choices, our intransitivites, may be a precisely calibrated feature. The finite precision of our decision-making architecture may be efficient in the sense that reducing intransitivity any further might incur more metabolic cost than it yields benefit.

In fact, many of the technical irrationalities cataloged at the end of the $20^{\text {th }}$ century now seem to reflect an evolutionary trade-off that efficiently balances these costs-of-computational-precision against the costs-of-intransitivity (Steverson et al, 2019; Heng et al, 2020). Configurations of choice that at first seem problematic or puzzling, begin to look like they maximize the efficient trade-off of costs and benefits. Such systems may be seen as rationally imprecise, resting on a razors-edge balance that regulates inconsistency to achieve efficiency.

\section{From Multiple-Selves to Subjective Value}

The reigning supposition that human decision-makers are inconsistent because of an internal conflict is at least as old as literature. In the Phaedrus, Plato (2005) explains that we are like a charioteer with two horses, one "good" and one "emotional." Freud's system of id, ego and super-ego (Freud, 1923:1990), follows this script closely, and its adoption by Paul Maclean in his Triune Brain Theory (1973) firmly established the notion that multiple neural modules compete to control behavior. Data gathered in the last two decades, however, challenges this conclusion. Neurobiological searches for these competing modules have repeatedly identified instead a single representation of value. Although puzzling, the source of our intransitivities seems to be something more fundamental than an internal competition between our emotional and logical selves. 
The origins of our contemporary notion that value, subjective or objective, is represented neurobiologically in the form of a single common currency, has its origins in the first efforts to search for subjective value representations in the brain (Fig 1a; eg., Glimcher and Platt, 1999; Sugrue at al, 2004). Those studies identified what looked like a decision-variable, but it was not until 2004 that it was definitively shown that firing rates could be observed in the parietal cortex that tightly correlated with the economic notion of expected utility, but not at all with choice probability (Fig $1 \mathrm{~b}$; see also Louie at al, 2010). This evidence (Fig 1c) was silent however, about how these representations behaved when choosers became inconsistent. In order to search for conflict in the utility representation, one needed a reliably inconsistent behavior. For that, a number of labs turned to choices in which decision-makers are conflicted between taking less now or waiting patiently for more later, intertemporal choices.

Decision-makers prefer to receive rewards sooner rather than later. For economists there is nothing inconsistent about such a preference (Strotz, 1955). As long as the decision-maker decrements the subjective value of a reward consistently, one can prove that perfect transitivity can be maintained (Fishburn and Rubenstein, 1982). Unfortunately, neither humans nor animals seem to decrement reward value in this way. Instead discount rates are higher for rewards offered at short delays and lower for rewards offered at longer delays (e.g., Mazur, 1984; Ainslie, 1991; Myerson and Green, 1995).

How can one make sense of such inconsistent behavior? One answer (Laibson, 1997) drawn from the multiple-selves model is that our brains have two independent modules: An "impulsive" chooser which only values immediately available rewards and a "rational" patient chooser who values all rewards as a function of the delay-to-reward duration. In this conception, human behavior represents the combined action of these two modules - each internally consistent when acting alone - but when combined yielding strongly intransitive behavior.

In a landmark fMRI study designed to test this hypothesis (McClure et al, 2004) human decision-makers made real choices between rewards offered at different delays. When rewards were offered immediately a single value-related signal was identified at two locations: the ventral striatum (VS) and the ventromedial prefrontal cortex (vmPFC). When rewards were offered at longer delays, no value related signal was observed (Fig 2a). Either this was because immediately available rewards are just worth more and thus easier to detect with fMRI or it was because a specific impulsive module had been identified. To resolve that issue the authors set out to identify some signature of the patient chooser: Isolating the trials on which subjects took longest to respond, they looked for particularly active brain areas. They found evidence of such activation in the dorsolateral prefrontal cortex (dIPFC) and the PPC, and hypothesized that this might reflect activity of the independent patient module predicted by theory (Fig $2 b$ ). 
The finding, however, was unavoidably ambiguous. Was the VS signal so strong for immediately available rewards because it only encoded immediate rewards, or was it also encoding the subjective value of long-delayed rewards, but at a level so low that it was undetectable to early fMRI scanners. To answer one would need to examine activity in the VS and the VmPFC when the magnitude of the delayed reward was suddenly increased (Kable and Glimcher, 2007). This manipulation revealed that as soon as the delayed reward was large enough to yield a subjective value signal detectable by fMRI, its representation was clear in both of these brain areas: This was, it turned out, not an area that specifically represented the preferences of an emotionalimpulsive self, it was an area that represented all rewards, at a magnitude modulated by delay.

A detailed characterization of the VMPFC and VS activity revealed that the signal precisely captured, at a within-subject level, both the impulsive early high valuation and the more gradual later decline in value with time. The data revealed that this single brain activation was just as intransitive as the individual, even an individual subject's degree of inconsistency could be accurately derived from the activation pattern in either of these brain areas (Kable and Glimcher, 2007, 2010). Even when decision-makers were behaving inconsistently, there was a unitary neural representation of a single hidden decision variable (Figure 2c).

Subsequent replication of these experiments in monkeys (Figure 2d) shows, if anything, the match between the neural firing rates in the PPC and the irrational subjective value curves is even better at the higher resolution of single neuron recordings. Could it be that that there was a unitary representation of a single utility-like signal, rather than the many competing signals predicted by multiple-selves theory?

At this same time, a similar examination of another behavioral pattern sometimes seen as an inconsistency, loss aversion, yielded very similar results (Tom et al, 2007). Human subjects often treat losses relative to the reference point as more consequential than equivalent gains, presumably because losses are more anxiety-provoking. In this study brain activity was examined as decision-makers evaluated financial lotteries that involved varying levels of gains and losses. Just as in intertemporal choice, these data revealed that human choosers were inconsistent, but that this inconsistency seemed not to arise from multiple modules, one only anxious and one only logical. Instead the study revealed that a number of brain areas irrationally encoded losses and gains asymmetrically, but not by employing multiple independent modules (But see Knutson for one counter-example.)

A series of three subsequent meta-studies have very strongly supported this conclusion that a single decision-variable is represented neurally - that emotional and rational evaluations are fully integrated. (Levy and Glimcher, 2012; Bartra et al, 2013; Clithero and Rangel, 2014). All three of these metastudies, together aggregating data from hundreds of studies, tell the same story. Value representations in the brain do not 
reflect multiple competing agents. Instead, these data suggest that the irrationalities we observe in behavior reflect a fundamental irrationality in the neural representation of subjective value, in humans, non-human primates (Chen et al., 2006), in rodents (Constantinople et al., 2019), maybe even in insects (Shafir, 1994). The representation of subjective value seems fundamentally imperfect, but imperfect in similar ways in animals separated by hundreds of millions of years of evolution.

\section{From Irrational Subjective Value to Efficient Subjective Value}

What neural features underlie these imperfect or technically irrational representations of subjective value? One widely-accepted answer is that these imperfections arise from "unavoidable" limits to neural computation: Inconsistent behavior reveals an imprecision in the neurobiological representation that evolution has for some reason failed to eliminate (eg., Shadlen and Newsome, 1994; Mazurek and Shadlen, 2002).

Consider thinking about this process another way: The human brain consumes 10 watts of power. To provide that power, humans ingest $22000 \mathrm{kcal}$ of food a day, $20 \%$ of which (400kcal) drives the brain (Raichle and Gusnard, 2002). Imagine a brain with $10 x$ the precision and capacity, hence 10x the metabolic demand. That brain would require an additional $3,600 \mathrm{kcal}$, requiring that we triple our daily intake to support this $10 x$ increase in precision.

Should natural selection push against costly irrationalities towards a more precise brain, even if that added precision comes at such a high cost? Natural selection does not aspire to perfection. It pushes organisms towards greater fitness (eg. Hamilton, 1964). Building a brain which always picks the best candy bar is better than building a brain that occasionally picks second-best, but perhaps not if you have to buy three times as many candy bars to survive. Formally, it is known that precision is monotonically costly and increasing precision must, by the laws of thermodynamics, increase metabolic costs (Steverson et al, 2019). Once we incorporate these costs into our thinking, it becomes clear that intransitivities might simply be desirable economies. Rather than modeling neural decision-variables as Platonic signals corrupted by noise, one might see in them an optimized trade-off between informational density and representational costs. Once we acknowledge that it is efficiency, not rationality as the neoclassicists proposed, that the brain should maximize, this significantly changes how we need to model choice (Polania et al., 2019; Heng et al., 2020).

\section{When are Irrationalities Efficient?}

Random Utility Theory (McFadden, 1974) pioneered our understanding of the irrationalities expected from a chooser with limited precision. As precision declines, decision-makers make more and more errors, sometimes picking the second-best option rather than the best, or even occasionally picking the third-best option. The 
theory predicts that these errors are more common when the options being compared are very close in value, regardless of the values of those options. Classical Random Utility theory thus presumes that people are imperfect choosers, but it hypothesizes that there is no real pattern to their errors, certainly not patterns like the systematic irrationalities that we observe in human and animal choice behavior.

Could a biological system do better than Random Utility theory predicts? Consider an idealized chooser who knows she has limited precision to encode subjective value, but also knows with certainty that she is more than much, much more likely to encounter a subjective value in a particular range, for example something around the value of a candy bar rather than the value of a car (Fig 3A). Such a chooser would maximize their efficiency by dedicating more precision to the likely range of subjective values and less to the unlikely ranges; efficiently adapting their precision to maximize accuracy where it is most important and to minimize accuracy where it is less valuable (Fig 3B). This efficient coding hypothesis (Box 3), the idea that neural codes could be efficient, not simply accurate, was first studied in the 1950s and 60s (Attneave, 1954; Barlow, 1961) and has been broadly impactful in the study of perceptual systems (Reynolds and Heeger, 2009).

Consider a chooser who has to represent the nutritional value of food choices so as to maximize expected nutritional outcomes. We can think of Figure $3 \mathrm{~A}$ as plotting a histogram of the many options that our chooser might encounter in a particular environment (Laughlin, 1981). For this one-dimensional case the optimal encoding strategy for a system of limited precision - the provably efficient solution - is for the neuronal firing rate function to be the integral of the frequency histogram (Figure 3C). For contrast, consider Figure 3D which plots a linear firing rate function. Of course, like all neurons these two hypothetical neurons are imprecise. Their mean firing rate is marked by the black line, but moment-by-moment firing rates lie randomly within the gray bands. Notice that when the efficient neuron is firing at half its maximal rate, we know that reward magnitude lies between 13 and 17 . In contrast, when the linear neuron is at half maximal rate the reward magnitude lies between 20 and 43 . This reveals that by tuning the firing rate function correctly, we can increase precision where it is most valuable at the cost of decreasing precision where it is least valuable. Of course, this would also impose a structure on our errors, intransitivities would be more common in some cases than others.

\section{The Theory of Efficient Representation}

A significant amount of work in the 1990s (Heeger, 1993; Heeger et al., 1996; Simoncelli and Heeger, 1998) examined efficient coding in the visual system using the Divisive Normalization Equation:

$$
F R_{1}=\frac{I n p u t_{1}^{\alpha}}{M+\sum_{i} \omega_{i} \text { Input }_{i}^{\alpha}} \quad \text { eq } 1
$$


Where the firing rate of neuron 1 is equal to the primary input to that neuron (for example the brightness of a particular pixel) raised to an exponent divided by the Mode (or center) of the input distribution plus a weighted sum of all current inputs to the other neurons, each raised to the same power.

Using numerical methods (Schwartz and Simoncelli, 2001) it has been shown that if this divisive normalization equation is used to represent a set of visual images pixel-by-pixel, one can find values for $\alpha, M$ and $\omega$ that do, in fact, minimize the total number of errors (or equivalently maximize the amount of information) in the representation of the image. This class of function re-encodes visual images in a way that is maximally efficient, putting the most information in the fewest action potentials. In the last few years, this work has been extended to the domain of subjective value (Steverson et al, 2019) and has yielded a clear view of how an efficient decisionmaking system would encode subjective value and thus what kinds of intransitivites it would be expected to produce. Treating the efficient encoding problem as a problem in thermodynamics, it was possible to derive the class of functions that achieves an optimal tradeoff between maximizing information and minimizing costs. Interestingly, the class of functions derived in this way could be shown to be formally equivalent to the divisive normalization class of representations used in the study of the visual system. Perhaps even more surprising, a huge class of behaviorally observed "irrational" choice behaviors can also be shown to be mathematically equivalent to both divisive normalization and to information maximization. Divisive normalization class functions are optimally efficient representations for subjective value, and they unavoidably yield irrational choice behavior specifically because the efficiently trade-off the costs and benefits of precision.

So what exact firing rate functions (or equivalently subjective value functions) should we expect to see in an efficient system? A number of groups (Polonia et al., 2019; Heng et al., 2020; Tajima et al., 2019) have examined the problem of describing how a neural system might be designed to optimally encode specific inputs. The basic answer is that an optimal system must select a transformation function that maximizes a specific quantity (for example, calories obtained) for a specific class of inputs. The firing rate function needs to be tuned to the inputs just as in Figure 3a. This work is critical because it refocuses us on two properties that are the subject of intense current investigation: What is the input distribution the chooser should seek to represent and what is the actual object (expected information, expected value or expected sample recovery) being maximized. All of this work, however, points at the notion that in order to maximize fitness, organisms require economical transformations of value that yield some irrationalities because eliminating those irrationalities would be inefficient. 


\section{Divisive Normalization in Choice}

The classic divisive normalization equations achieve efficiency by devoting more firing rate range to more likely inputs. These functions tune their responses with two key parameters, $M$ and alpha. As Figure 4 shows for a simple example with a single choice option, $\mathrm{M}$ centers the transformation on the most likely input and alpha controls the overall shape of the function. Notice that at alpha $=1$, the function looks very much like a classical utility function (Bernoulli 1738: 1954). As alpha increases, that function takes on the characteristically sigmoidal shape of the Kahneman and Tversky (1979) value function. This suggests that the shift from a classically concave function to a sigmoid observed behaviorally may be driven by changes in the distribution of the options under consideration.

It is worth noting that in situations with more than one option, as in equation 1, M serves as a kind of a temporal average, an estimate of what future input is most likely. The weighted sum which aggregates information about the current options, in contrast, is a kind of spatial average that, because of the exponent, controls the shape of the function based on the specific values of each the current inputs. These temporal and spatial averages can be thought of as a kind of prior and likelihood estimation, maximizing information in the posterior firing rate distribution (Steverson et al., 2019). In fact, some current research suggests that the relative contributions of temporal and spatial components may be under dynamic control so as to better align the firing rate function with a best estimate of the input distribution (Zimmerman et al., 2018).

\section{Efficient Representation of the Most Common Input}

If $\mathrm{M}$ reflects a tool for centering the encoding function on the median or mode of the distribution of possible rewards, then what happens if we change the size of the median (or modal) reward size? In 2005 this experiment was conducted while recording from midbrain dopamine neurons (Tobler et al., 2005). As predicted, dopamine neurons do indeed re-centered their firing rate functions in response to changes in the input distribution. More recently, similar results have been observed in the orbitofrontal cortex (Padoa-Schioppa, 2009) and the posterior parietal cortex (Louie et al., 2011). The neural firing rate functions do adapt to changes in the input distribution as expected in an efficient system. But what kind of irrationalities would such a system yield at the behavioral level (see also Conen and Padoa-Schioppa, 2019)?

In one experiment designed to search for such irrationalities (Khaw, Louie and Glimcher, 2017; Figure 5), hungry subjects were asked to indicate the maximum amount they would pay for each of 30 different snacks. Ten high value (and 10 low value) snacks were then identified for each individual, and subjects made repeated bids on these high (or low) value items during a 300-trial adaptation block. Without warning, the input set was suddenly changed to include all 30 snack foods. After the change, subjects transiently shifted their bids downwards (or upwards after low-value 
adaptation), and this shift decayed over the course of about 45 trials - exactly the effect that would be expected if an efficient coding system using a divisive normalized representation was re-centering the mode of its representation. The critical feature is that the subjects showed inconsistent pricing behavior when the mean of the input distribution changed. That inconsistency, however, reflected a provably efficient encoding scheme that makes the most of a limited informational capacity (See also Juechems et al., 2021).

\section{Efficient Representation of the Current Offerings}

The role of the weighted sum in the denominator is to adapt the firing rate function to the current choice set. Consider a situation in which all of the options in the current choice set are suddenly high-valued. In a system without divisive normalization, this can result in all of the firing rates being close to the maximum rate and thus hard to distinguish - a loss of information created by the bounded and limited precision firing rate. An efficient system solves this problem by reducing the overall firing rates (much like $\mathrm{M}$, but instantaneously), more accurately centering the firing rates for each option within the available firing rate range ${ }^{1}$.

\section{Choice Set Size Effects}

Such a system, however, would be highly sensitive to the number of options under consideration. Note that as the number of elements in the denominator grows, so does the aggregate value of the denominator, shifting overall firing rates lower and lower. As choice sets grow in size, error rates (irrationalities) should increase in a characteristic fashion - a pattern very different from the class of errors predicted by Random Utility Theory (Webb et al., 2020). This arises because as the number of options increases, the amount of information we put into the system also increases. Performance decays efficiently as the informational demand on the network increases.

It has been known for some time (eg. lyengar and Lepper, 2000) that as choice sets increase in size, performance decreases. A precise examination of the form of performance decrease observed under these conditions, however, reveals that the divisive normalization model significantly outperforms traditional random utility models in accounting for the irrational features of decision-making in the face of large choice sets. While humans do choose irrationally when faced with large choice sets, it appears they do so in a way that maximizes accuracy in the face of increasing informational demands.

\section{Three Option Irrationalities}

A more subtle class of irrationalities that one might expect in an efficient system are so called- three option errors, errors that arise when subject must identify the best of

\footnotetext{
${ }^{1}$ Formally, omega reduces informational redundancy induced by input covariance, alpha captures distributional shape, and then the summing operation adjusts the width and lateral position of the function appropriately.
} 
three possible options (eg., Louie et al., 2011; Li et al., 2018). Consider the problem faced by a divisively normalized system when it must choose between two options very close in value to one another, both in the presence and in the absence of a low-valued third option. The insertion of the third option, even if it is never chosen, adds information to the system and should degrade performance. Worse, as the value of the unchosen third option increases, performance (and neuronal firing rates) should degrade further. Parietal neurons (Louie, Grattan and Glimcher, 2011) turn out to show patterns of firing rates that align well with the prediction of the divisive normalization model under these conditions as well. Subsequent work ( $\mathrm{Li}$ et al, 2018) aimed at understanding the role of gain adaptation more generally on encoding accuracy has yielded a similar result. $A$ generally efficient representation could account for systematic errors as efficiencies, in this case under a broader range of conditions than those captured in the original experiment.

It should be noted, however, that this class of three-option irrationalities has been somewhat less controversial than some of the other features of an efficient representation which have bene more widely observed. One important paper (Chau et al., 2014) yielded similar results, but not with complete consistency. Another study (Gluth et al, 2020) initially reported a failure to observe this particular irrationality in human choosers when measuring reaction times rather than choices, although a reanalysis of that data (Webb et al., 2020) suggests that the majority of their subjects do show this effect, if somewhat more weakly than might have been expected. While much work remains, it does seem clear that richer forms of divisive normalization (Landry and Webb, 2021) will be important and may be able to effectively capture even these more heterogeneous results.

To summarize, efficient coding systems should show very characteristic irrationalities, and there is growing evidence that the neural architecture is well tuned to provide just this kind of efficiency (Box 4). Choice errors should show a strong dependency on a kind of reference point, and they do both neurally and behaviorally. Subjects should be risk averse above this reference point and risk seeking below it, as has been observed. Sudden shifts in the mean of the input distribution should cause systematic intransitivities (like the ones Nick Leeson showed) while the reference point is adapting, again as observed. Increases in the number of options should lead to inconsistencies as should direct manipulation of the structure of the choice set, a phenomenon that has now been well documented. What should stand out here is that many of the inconsistencies that motivated Simon, Kahneman, Tversky, and the prospect theory they developed, emerge as efficient responses by an informationally constrained system. 


\section{From Expected Value Theory to Rational Imprecision}

In the mid-17 $17^{\text {th }}$ century Pascal proposed that what humans were doing when they made choices was to objectively maximize expected value. The goal of choice should be defined by a simple objective function: Compare options by multiplying the probability of each outcome with the magnitude of gain and then select the option that objectively maximizes the long-run average value one accumulates from all of one's decisions. Clearly Nick Leeson was not doing that when he bet his bank on riskier and riskier options each day.

As it became clear that human choosers often fail to maximize expected value, mathematicians abandoned the notion of an objective maximization function. Instead they concluded that humans maximize a hidden subjective transformation of value which today we call the utility function. The great virtue of this approach is that it appears to explain both why and how humans are risk averse.

The neoclassical economists of the last century shifted their emphasis away from the specification of a particular utility function and towards a more general notion of what constituted utility maximization. Developing the notion of rationality, they defined axiomatic rules that any kind of perfect maximizing behavior would have to follow. If humans followed these rules, humans were choosing in a goal-directed fashion even if we could not be certain exactly what goal or goals they were pursuing.

Accepting that logic, empirical economists and psychologists working at the end of the $20^{\text {th }}$ century noted a wide range of conditions under which humans behaved intransitively. While a few economists and psychologists sought to explain that this behavior might be related to the physical limitations of human brains, there was an overwhelming shift towards seeing human choosers as accumulations of small mechanisms that were troublingly inefficient when working in aggregate. Instead of trying to define why humans chose the way that they did, these scholars tended to accept that humans were disordered.

Interdisciplinary work conducted over the last two decades, however, offers a us the possibility of a very different interpretation. These data seem to suggest that, as Pascal might have suggested if he had been a neurobiologist, humans really do employ a representation of the expected value of each option, but a representation distorted by the needs of a limited precision neural representation. What these data seem to show is that our biological objective function really is, as Pascal hoped, to maximize long-run average value. But this is a maximization we wisely perform with a level of precision driven by the constraints of efficiency. In this view, the structure of the transform from value to subjective value is specified not by a need for risk aversion, as the classical economists of the $19^{\text {th }}$ century imagined (eg., Mill, 1863), but by a need for efficiency. We can conclude instead that the observed subjective value function reflects a tradeoff between the rising cost of precision, the diminishing returns from increasing precision, and the distributional properties of the world in which we make choices. 
What is perhaps most ironic about that notion is that Pascal may have been less wrong than we have thought for the last three hundred and fifty years. The utility function is not be so much a moral value as Bernoulli proposed in the $18^{\text {th }}$ century, but rather a mechanistic tool that tunes our behavior for maximal efficiency in the face of limited precision.

\section{Concluding Remarks}

The framework we use to make sense of human choice behavior impacts how we think about ourselves and the predictions we make about how people will choose in novel situations. This influences not just research, but also social policy. As our understanding of human behavior has changed, the policies we consider fair or desirable also change.

In the 1950s humans were thought of as perfectly rational. People accurately maximized their personal preferences, or utility functions. To limit an individual's choices, or to force a particular choice, was unthinkable (as long as no one else was being harmed).

In the 1970s and 1980s, however, it became clear that humans are often irrational, apparently beset by unresolvable internal conflict between their multiple selves. Policy makers could help such a decision-maker by restricting their options so as to minimizes destructive internal conflict. This logic, that irrationalities arise from an internal conflict and that policy makers should structure the choice environment to eliminate these conflicts, has recently taken a dominant role in policy circles (Thaler and Sunstein, 2003). Designing the choice environment to limit conflict inside the decisionmaker is now a standard recipe for improving the wellbeing of decision-makers.

The neuroeconomic data presented here, however, seem to indicate that humans are not intransitive because of internal conflict. Instead, these data suggest that human intransitivites arise from the limits of human computational precision and how the brain distributes that precision in representing subjective value. If true, that conclusion calls for policy interventions that may be quite different from the ones prescribed by contemporary behavioral economists. In an efficiently irrational chooser, eliminating irrationality ultimately comes down to finding ways to reduce either the costs, or the need for, precision. Consider this example: Human choosers are intransitive (Khaw et al., 2017) when they rapidly shift from choosing at a low value range to a high value range (Figure 5). This reflects not a conflict between two modules, but rather the slow adaptation of the divisive normalization mechanism as it shifts to maximize precision in a new range. In this case, minimizing irrationalities is simply a matter of waiting for the mechanism to align with the new value range.

All of this suggests that our new understanding of the limits of the choice mechanism reveal a surprisingly simple structure that admits new approaches to old 
irrationalities. Understanding that irrationalities emerge from the limits of our precision will suggest new classes of policy interventions that specifically target our representational limits while exploiting our irrational efficiencies. 
Figure 1: Parietal neurons carry an abstract representation of subjective value

Value Representation in Parietal Cortex

A. Firing Rate Correlates with Value and Probability
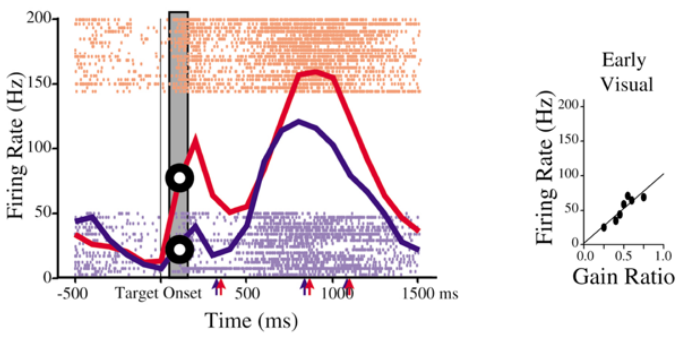

B. Not With Choice Probability

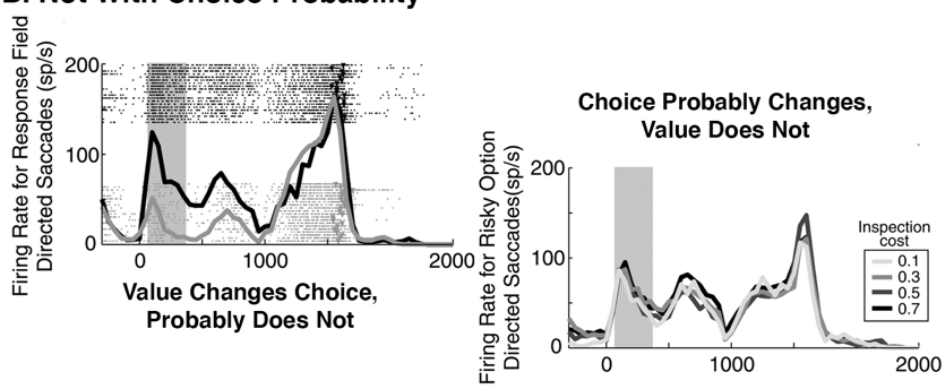

A. Firing Rate Tracks Dynamically Changing Value

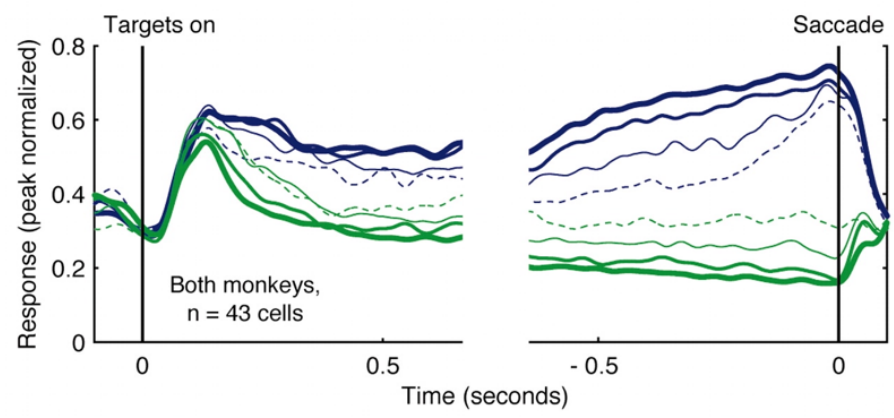

Figure 1. Value Representation in single neurons. A. When awake behaving monkeys are presented with potential rewards, the activity of single neurons encodes the value of those rewards. Parallel experiments (not shown) indicate that when the probability of reward is varied, these same neurons also encode reward probability in exactly the manner predicted by economic theory. (Platt and Glimcher, 1999) B. These changes in neuronal activity specifically encode reward value and not the likelihood that a decisionmaker will select the reward. Using a strategic game, it is possible to show that when reward magnitude varies but choice probability is held constant (left) these neuronal firing rates change. When reward value is held constant but choice probabilities are systematically varied (right panel) the neuronal firing rate is constant. (Dorris and Glimcher, 2005) C. When reward rates are dynamically changed and the decisionmaker must decide how to best allocate its choices to maximize reward, neuronal firing 
rates depend on average rate of reward. Stacked red and blue lines accurately rank rates of reward actually obtained by freely choosing monkeys. (Sugrue, Corrado and Newsome, 2004) 
Figure2: Human subjective value signals predict both impulsive and patient choices

A. BOLD Responds Strongly For Immediate Rewards

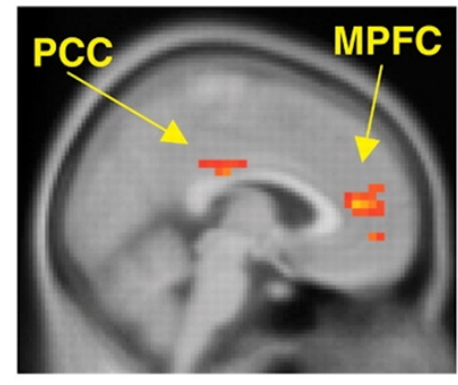

$x=4 \mathrm{~mm}$

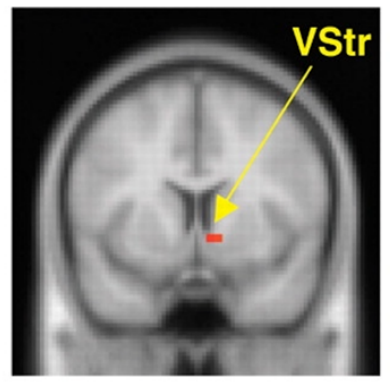

$y=8 m m$

B. BOLD Tracks Subjective Value at All Delays
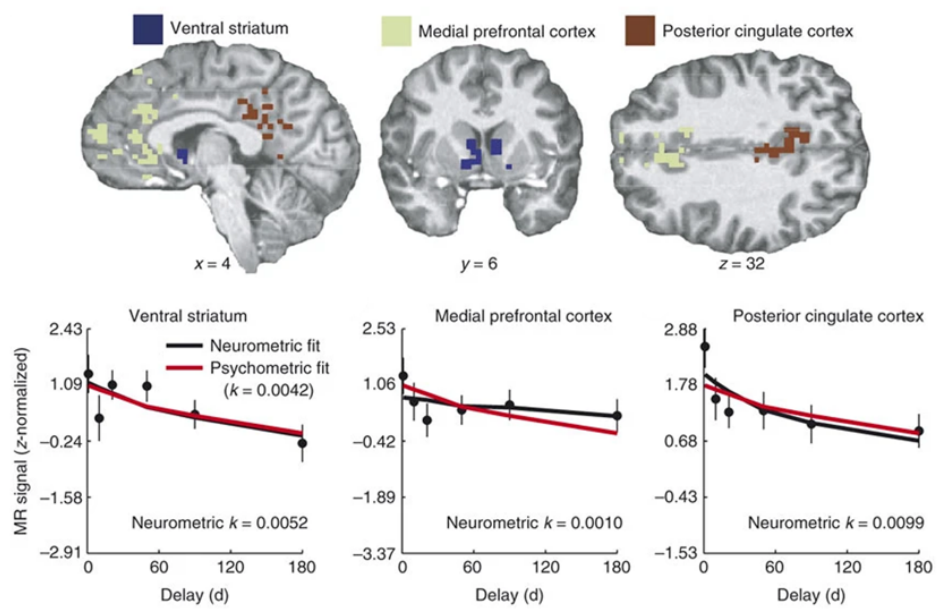

C. Neuronal Activity Tracks Subjective Value at All Delays
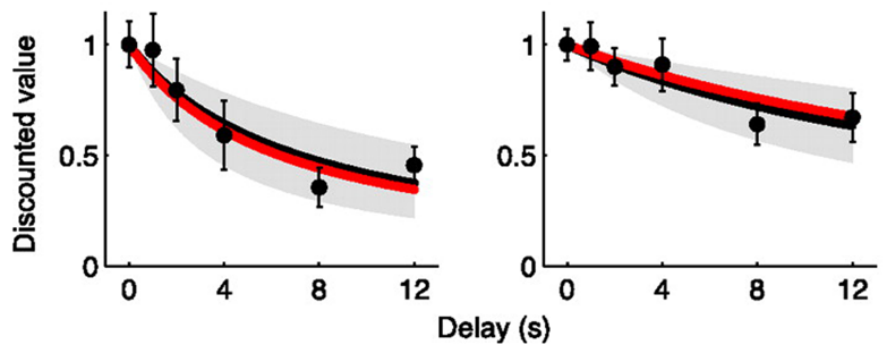

Figure 2. Value-related brain activity tracks subjectively measured reward magnitude in an intertemporal task, even when that subjective value declines hyperbolically in a way characteristic of inconsistent choices. A. Activity in the MPFC, PCC, and VStr is much stronger for immediately available rewards than it is for delayed rewards. (McClure et al, 2005) B. For a single representative subject, activity in these areas declines smoothly (dots), but hyperbolically (black curves), as the delay to reward increases. This decline precisely matches the same subject's behaviorally estimated subjective value curve, which is also hyperbolic (red). (Kable and Glimcher, 2007) C. More precise measures of 
single neuron activity made in monkeys making intertemporal choices yield even more precise matches between behavioral estimates and neuronal estimates (same colors as in B). Both behavioral and neural estimates are clearly hyperbolic. 
Figure 3: Noisy neural systems must adopt non-linear firing rates to be efficient
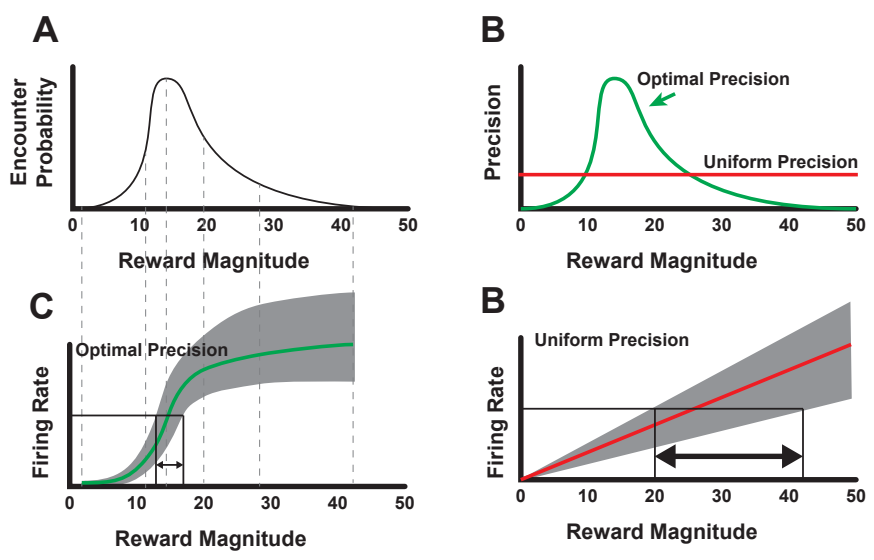

Figure 3. The goal of efficient coding in decision-making is to maximize information about the objective values of rewards in the spike rates that will be used to compare options. This maximizes the likelihood of selecting the best possible option. A. Hypothetical distribution describing the objective probability that a chooser operating in an example environment will encounter rewards of any given magnitude. $B$. To maximize information precision should be greatest at reward magnitudes that are likely to be encountered. Allocating precision uniformly erroneously dedicates neuronal bandwidth to options which are rarely encountered. C. Taking the integral of the encounter probability curve yields (under 1-dimenstional conditions) a firing rate function that maximizes accuracy at the expectation point. The half-maximal firing rate (black horizontal line) then indicates the actual reward magnitude with higher precision. D. A uniform firing rate, in contrast, yields estimates of reward magnitude that are less accurate at expectation (and more accurate off expectation). 
Figure 4: Divisive normalization firing rate functions are highly flexible
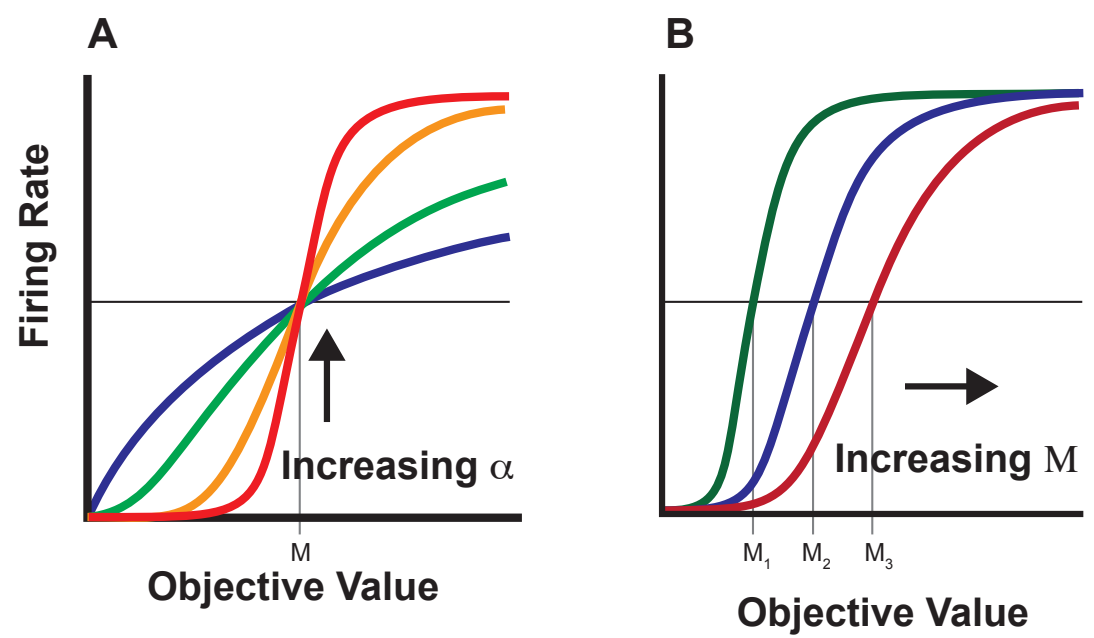

Figure 4. Functions that relate firing rate to objective reward magnitude for a system employing divisive normalization (eq 1 in main text. Note that in this functional form derived from Tymula and Glimcher, 2021, the exponent alpha is embedded in the constant $\mathrm{M}$, rather than being written separately for clarity in expressing the lateral shift of the function.). A. Adjusting the exponent in the equation steepens the function around the expectation, or reference, point. This corresponds to adjusting the firing rate function to encode narrower or wider input distributions. Note, however, that these functional shapes also tile the set of previously described utility and value functions. When alpha = 1 (blue) the function mimics the shape of the classical utility function proposed by 
Bernoulli. As the exponent increases (red, alpha $=3$ ), the function comes to resemble the value function of Kahneman and Tversky. $B$. As the expectation point, $M$, is increased, the center of the function shifts to the right. This corresponds to a strategy that centers the firing rate function on the median value of the input distribution. 
Figure 5: Human choice anomalies show evidence of divisive normalization
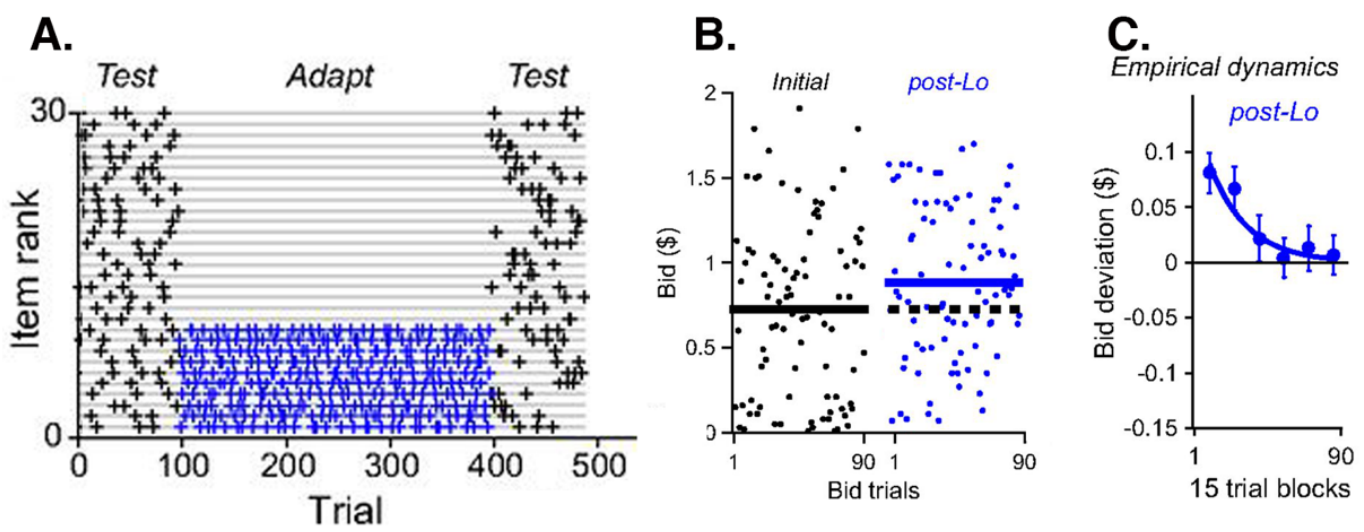

Figure 5. Effect of adapting the reference point on choice behavior. A. Task design from Khaw and colleagues. Subjects state the maximum amount they would pay for different foods in an incentive compatible manner. First, they encounter a range of values (black). Subjects are then adapted to a low value subset, shifting the expectation downward (blue). Finally, valuations are remeasured immediately after adaptation (black). B. Initial bids made during the first test period show an average value of about $\$ 0.70$ (black). Measurements during the second test period indicate that subjective values are initially shifted upwards by adaptation about $10 \%$ (blue). C. The effect of this adaptation of the reference point declines over 90 trials with a roughly exponential time course. 


\section{Box 1: Inconsistent Patterns of Choices}

Inconsistent patterns of choices are those in which the decisions of a chooser cannot be construed as pursuing a meaningful goal. A classic example would be a chooser who prefers apples to oranges and oranges to pears, but also prefers pears to apples (Figure la, Choice cycle). Such a chooser who had a pear should reasonably be expected to trade their pear plus one cent for an orange. Having gained the orange, we might reasonably expect them to trade that orange plus one cent for an apple. What makes this pattern inconsistent, is that such a chooser should then also be willing to trade their apple plus one cent for their original pear. At this stage they have spent money and time, but gained nothing - a classic definition of an inconsistent choice.

Broadly speaking, patterns of inconsistency can be separated into two classes: Random and Structured. Random inconsistency arises when subjective value varies randomly from moment to moment. A decision-maker who, on average, values apples only a tiny bit more than oranges would occasionally make an intransitive choice just due to these uniformly distributed random fluctuations (Figure 1b). These kinds of inconsistencies have been well-characterized by Random Utility Models (McFadden, 1972; Gul and Pesendorfer, 2006). The key feature of this class of model is that intransitive errors are expected to be distributed uniformly across the whole range of subjective value.

Of greater relevance to this article are patterns of inconsistencies that suggest a non-uniform distribution of intransitive errors. A chooser who prefers $\$ 3$ for sure over a $50 \%$ chance of winning $\$ 10$ when in the presence of $50 \%$ chance of winning $\$ 3$, but prefers the risky $50 \%$ chance of winning $\$ 10$ when the unselected lottery is removed, shows a more structured pattern of inconsistency. Structured inconsistencies of this kind generally arise from context effects; changing the context in which an offer is made systematically changes the decision-maker's preferences in a systematic and repeatable way.

The best understood of these context effects are those mediated by the reference point. One of Kahneman and Tversky's (1979) central observations was that whether a chooser was risk-seeking or risk-averse depends not merely on the specific outcome they are considering but also on a hidden internal reference point (originally called the status quo). Shifting the hidden reference point in a systematic way can can even lead to choice cycles of the kind described above.

In general, inconsistencies associated with Random Utility Theory and the more complex inconsistencies associated with behavioral economics have been seen as distinct. One central goal of Rational Imprecision Theory (Steverson et al, 2019) has been to provide a framework which unites these two classes of inconsistency by defining non-uniform random utility models that can capture both classes of inconsistent behavior. The main text of this article suggests a resolution to many classes of inconsistent choice in this way. 
Figure I: Patterns of intransitive choice

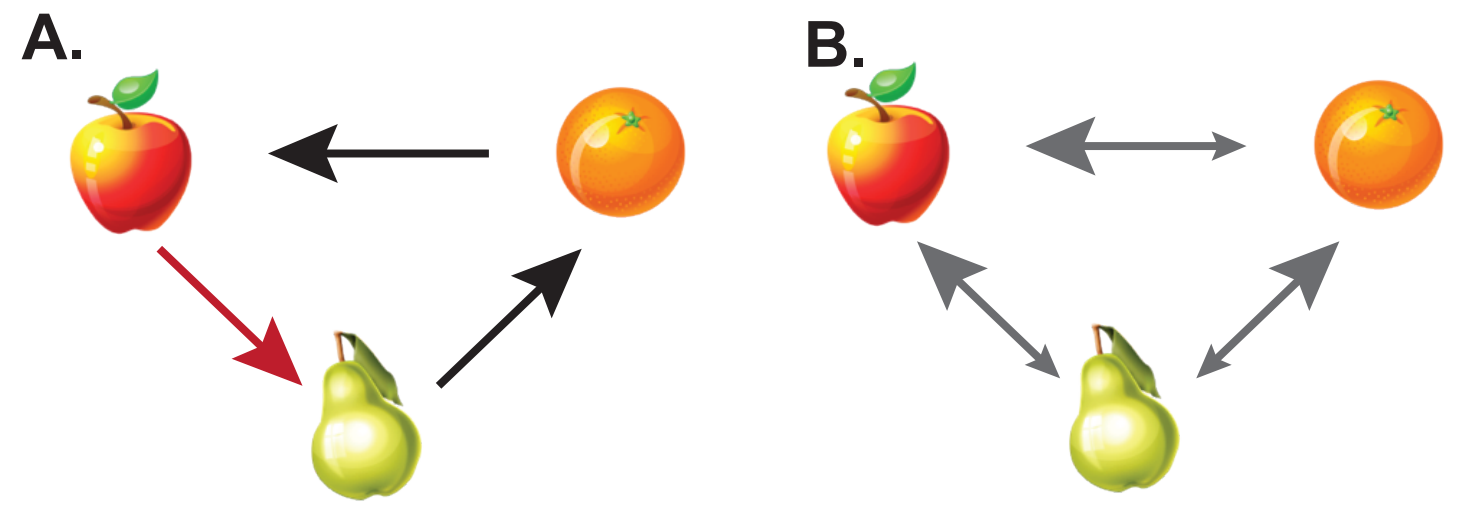




\section{Box 2: Rational Choice}

The word "rationality," as used by economists, has a highly technical meaning and this has generated endless confusion among non-economists. At its heart, the notion captured by the phrase rational choice is the idea that "rational choices" are those that show logical consistency. This idea was first developed in the 1920s in an effort to identify patterns of choice that were objectively wasteful.

What can be said about a chooser who strictly prefers apples to oranges but also strictly prefers oranges to apples. Samuelson (1937) showed that there is no possible mathematical transformation of value (or anything related to value) that can be maximized by such a pattern of behavior. Such a chooser is defined by an economist as "irrational" because they their choices are not directed at any kind of maximization, their choices cannot be said to be goal directed.

The 1940s saw an expanded mathematical definition of rationality that was somewhat more positivist. Houthakker (1950) examined the logical properties of a chooser who is both transitive in their choices (they prefer apples to oranges to pears) and who is monotonic in their preferences, they never see more of a good thing as worse than less of a good thing. He proved that such a chooser can always be described as maximizing some monotonic transformation of value, they are always by definition: technically rational. Thus, a decision-maker who has a consistent plan to maximize utility by purchasing drugs rather than food, would be identified by an economist as "rational" in the technical sense (Becker and Murphy, 1988). In contrast, someone who switches back and forth between maximizing their drug consumption and maximizing their food consumption would be technically defined as irrational.

The important thing to remember when thinking about the economic notion of rationality is that it is entirely neutral with regard to morality, ethics, and perhaps even to some intuitive notions of well-being. It is perfectly reasonable to say that someone is rational in their pursuit of heroin, suicide, or world domination. All that rationality defines is the internal consistency, the goal directedness, of the choices they use to pursue those goals. 


\section{Box 3: The Efficient Coding Hypothesis}

Attneave (1954) and Barlow (1961), Building on Claude Shannon's Theory of Information (Shannon and Weaver, 1949), proposed that neural codes in sensory systems were driven by evolution towards maximization of the amount of information carried by each action potential. This information maximization can be viewed as a twostage process. First, eliminate redundancy in the incoming information. Second, adjust the firing rate function so more of the available range is dedicated to encoding likely inputs. This prevents one from wasting bandwidth on improbably events that are never observed.

Neurobiological functions that achieve information maximization when encoding visual images have now been widely studied (eg., Heeger et al., 1996; Schwartz and Simoncelli, 2001). Neurons in the visual cortex, for example, encode something like:

$$
F R_{1}=\frac{X_{1}{ }^{2}}{\sigma^{2}+\sum_{i}\left(\omega_{i} X_{i}\right)^{2}} \quad \quad \mathrm{Eq}
$$

where $\mathrm{FR}_{1}$ is the firing rate of the neuron encoding the objective intensity of pixel $1, \sigma$ is the expected mean intensity level, and $\omega_{\mathrm{i}}$ are weights that capture the correlations between pixels. The denominator removes from the firing rate of neuron 1 information already carried by other neurons, maximizing information per action potential. This model, known as divisive normalization, has subsequently been observed in many sensory systems and it has even been proposed that this mechanism is a canonical neural computation (Carandini and Heeger, 2011).

It has also been proposed (Glimcher, 2011) that a similar process might be operating in the neural representation of subjective value, a hypothesis that has been confirmed in a number of experiments (eg., Louie et al, 2014). While this is an active area of research, it is not yet clear exactly how efficiently either sensory or decisional variables are represented in the nervous system. In the next decade or so it should become clear whether neural computations like divisive normalization provide an accurate approximation widely used by the nervous system or whether more complex models will be required to understand the underlying neural architecture. 


\section{Box 4: Computing Subjective Value with Neurons}

One important test of any neurobiological theory is whether real neurons can perform the computations the theory requires. Growing evidence suggests that simple networks can indeed perform divisive normalization-like computations (LoFaro, 2014). In such a network (Figure II), neuron $R_{1}$ receives a direct excitatory input $\left(V_{1}\right)$, a baseline rate of activation that it shares with all other $R$ neurons, and a single inhibitory interneuron input, $G_{1}$, that divisively regulates $R_{1}$ 's activity. Each gain control neuron receives inputs from all other output neurons, thus computing a (weighted) sum of those inputs and using that as the divisive input to the output neurons exactly as required by the efficient equations. Although such broad connectivity seems counterintuitive, it has been documented in the rodent visual system (Sato et al., 2016).

Essentially all networks of this kind have a single equilibrium state (LoFaro et al, 2014). When inputs are applied to the network, the network eventually settles to represent the input divided by a weighted sum of the outputs of all of the output neurons. If the inputs change faster than the time constant of the network, the output signal represents a time-weighted average of the sequence of inputs. One cannot help but notice that this means that any broadly integrating gain control network converges to something almost like, but not completely identical to, the divisive normalization equations described in the main text.

At full equilibrium the output of the network shown in Figure II closely approximates:

$$
F R_{1}=\frac{x_{1}}{M+\sum_{i} \omega_{i} F R_{i}} \mathrm{Eq} 2
$$

How might $\mathrm{M}$ be represented in a real network? One possibility is long timeconstant version of this network might compute $\mathrm{M}$ and pass it on to a faster network optimized to compute the weighted sum of current options. When two such networks are cascaded, one with rapid dynamics and one with slow dynamics, the network with slow dynamics effectively computes the $\mathrm{M}$ term and then injects it into the faster network (Zimmerman et al, 2018).

At least in the parietal cortex, both the dynamics predicted by these models and the dependence of firing rate on output (rather than input as in the original divisive normalization equations) has now been observed (Louie et al, 2015). Parietal neurons do, indeed, receive both excitatory and inhibitory inputs and those inputs are, over at least a limited range, interacting roughly divisively in nature as required by the models. 
Figure II: A divisive normalization network

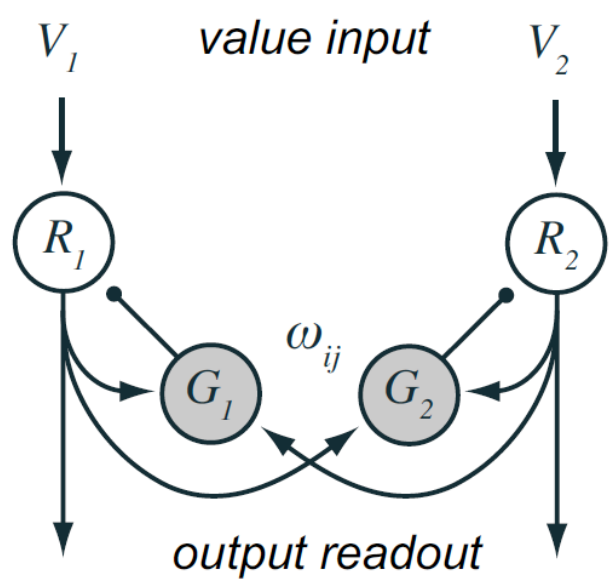

$$
\begin{aligned}
& \text { Excitatory neurons } \\
& \qquad \tau \frac{d R_{i}}{d t}=-R_{i}+\frac{V_{i}}{1+G_{i}}
\end{aligned}
$$

Inhibitory neurons

$$
\tau \frac{d G_{i}}{d t}=-G_{i}+\sum_{j=1}^{N} \omega_{i j} R_{j}
$$

The proposed network of LoFaro and colleagues which computes a divisive normalization-like transformation. Individual pyramidal cells receive an objective value input. Each R-neuron's output is divisively reduced by a nearby inhibitory interneuron that aggregates information about all inputs to the R-neuron layer. The G-neurons thus capture all available information about the choice set and remove that information from R-neuron, effectively maximizing information about how that neuron's $V$-input differs from other V-inputs. LoFaro and colleagues showed that models of this class must have a single equilibrium state which closely approximates standard divisive normalization. 


\section{References}

Ainslie, G. (1991). "Derivation of "rational" economic behavior from hyperbolic discount curves". American Economic Review. 81: 134-140.

Allais, M. (1953). Le comportement de l'homme rationel devant le risque. Critique des postulats et axiomes de l'ecole américaine [Rational behavior under risk: criticism of the postulates and axioms of the American school]. Econometrica, 21(4): 503-546.

Arnauld, A., and Nicole, P. (1662; 1996). La Logique, ou l'art de penser (Logic or the Art of Thinking), J.V. Buroker (trans. and ed.) Cambridge: Cambridge University Press, 1996.

Attneave, F. (1954). Some informational aspects of visual perception. Psychological Review, 61: 183-193.

Barlow, H. B. (1961). Possible principles underlying the transformation of sensory messages. In: W. A. Rosenblith (ed.), Sensory Communication. Cambridge, MA: MIT Press, pp. 217-234.

Bartra, O, McGuire, JT and Kable, JW. (2013) The valuation system: a coordinate-based metaanalysis of BOLD fMRI experiments examining neural correlates of subjective value. Neuroimage. 76: 412-427.

Becker, G.S. and Murphy, K.M. (1988) A theory of rational addiction. JPE 96: 675-700.

Bernoulli, D. (1738; 1954). Exposition of a new theory on the measurement of risk. Econometrica 22: 23-36.

Caplin, A, Dean, M, and Martin D. (2011) Search and Satisficing. AER. 101: 2899-2922.

Carandini, M. and Heeger, D.J. (2011) Normalization as a canonical neural computation. Nature Reviews Neuroscience. 13: 51-62.

Chau, BKH, Kolling, N, Hunt, LT, Walton, ME and Rushworth MFS. (2014) A neural mechanism underlying failure of optimal choice with multiple alternatives. Nature Neuroscience. 17: 463470.

Chen, M. K., Lakshminarayanan, V., and Santos, L. R. (2006). How basic are behavioral biases? Evidence from capuchin monkey trading behavior. Journal of Political Economy, 114(3): 517537.

Clithero, JA and Rangel, A. (2014) Informatic parcellation of the network involved in the computation of subjective value. 9: 1289-1302. 
Conen, KE and Padoa-Schioppa, C (2019) Partial Adaptation to the Value Range in the Macaque Orbitofrontal Cortex Journal of Neuroscience 39: 3498-3513 .

Constantinople CM, Piet AT, Brody CD (2019). An Analysis of Decision under Risk in Rats. Current Biology, 29:1-9.

Dorris, M. C., and Glimcher, P. W. (2004). Activity in posterior parietal cortex is correlated with the subjective desireability of an action. Neuron, 44: 365-378.

Fishburn, PC and Rubinstein, A. (1982) Time Preference. Intl Econ Rev 23: 677-694.

Freud, S. Strachey, J (ed)(1923:1990) The Ego and the Id. New York: Norton.

Glimcher, PW (2011) Foundations of Neuroeconomic Analysis. New York: Oxford.

Glimcher, PW and Fehr, E. (2013) Neuroeconomics: Decision-Making and the Brain. New York: Academic Press.

Gluth, S., Kern, N., Kortmann, M. \& Vitali, C. L. (2020) Value-based attention but not divisive normalization influences decisions with multiple alternatives. Nat. Hum. Behav. 4, 634-645.

Gul, F and Pesendorfer, W. (2006) Random expected utility. Econometrica. 74: 121-146.

Hamilton W. D. 1964. The genetical evolution of social behaviour I, II. J. Theor. Biol. 7, 1-52.

Heeger, D. J. (1993). Modeling simple-cell direction selectivity with normalized, half-squared, linear operators. Journal of Neurophysiology, 70(5): 1885-1898.

Heeger, D. J., Simoncelli, E. P., and Movshon, J. A. (1996). Computational models of cortical visual processing. Proceedings of the National Academy of Sciences USA, 93: 623-627.

Heng JA, Woodford M., and Polania R. (2020) Efficient sampling and noisy decisions. eLife. 9:e54962

Houthakker, H. S. (1950). Revealed preference and the utility function. Economica, 17: 159-174.

lyengar, S. S., and Lepper, M. R. (2000). When choice is demotivating: Can one desire too much of a good thing? Journal of Personality and Social Psychology, 79(6): 995-1006.

Juechems, K, Balaguer, J, Spitzer, B and Summerfield. C. (2021) Optimal utility and probability functions for agents with finite computational precision. Proceedings of the National Academy of Sciences 118: e2002232118. 
Kable, J. W., and Glimcher, P. W. (2007). The neural correlates of subjective value during intertemporal choice. Nature Neuroscience, 10(12): 1625-1633.

Kable, J. W., and Glimcher, P. W. (2010) An "as soon as possible" effect in human intertemporal decision making: behavioral evidence and neural mechanisms. J Neurophysiol. 103: 2513-2531.

Kahneman, D., and Tversky, A. (1979). Prospect theory: An analysis of decision under risk. Econometrica, 47(2): 263-291.

Kahneman, D., Slovic, P., and Tversky, A. (1982). Judgement Under Uncertainty: Heuristics and Biases. New York: Cambridge University Press.

Khaw, MW, Glimcher, PW and Louie, K. (2017) Value adaptation via divisive normalization. Proceedings of the National Academy of Sciences. 114: 12696-12701.

Khaw, MW, Li, Z, and Woodford, M. (2021) Cognitive imprecision and small-stakes risk aversion. REStud 88: 1979-2013.

Kőszegi, B., and Rabin, M. (2006). A model of reference-dependent preferences. Quarterly Journal of Economics, 121(4): 1133-1165.

Kőszegi, B., and Rabin, M. (2007). Reference-dependent risk attitudes. American Economic Review, 97(4): 1047-1073.

Laibson, D. (1997). Golden eggs and hyperbolic discounting. Quarterly Journal of Economics, 62: 443-477.

Landry, P and Webb, R (2021) Pairwise normalization: A neuroeconomic theory of maultiattribute choice. Journal of Economic Theory. 193: 105221.

Levy, D.J., Glimcher, P.W. (2012). The Root of All Value: a neural common currency for choice. Current Opinion in Neurobiology. 22: 1027-1038.

Levy, I., Lazzaro, S., Rutledge, R.B., \& Glimcher, P.W. (2011). Choice from non-choice: Predicting consumer preferences from blood oxygenation level-dependent signals obtained during passive viewing. Journal of Neuroscience, 31: 118-125

Li, Vickie \& Michael, Elizabeth \& Balaguer, Jan \& Castañón, Santiago \& Summerfield, Christopher. (2018). Gain control explains the effect of distraction in human perceptual, cognitive, and economic decision making. Proceedings of the National Academy of Sciences. 115. 201805224. 10.1073/pnas.1805224115.

LoFaro, T., Louie, K., Webb, R., Glimcher, P.W. (2014) The Temporal Dynamics of Cortical Normalization Models of Decision-making. Letters in Biomathematics. 1: 209-220. 
Louie K., Grattan L.E., \& Glimcher, P.W. (2011). Reward value-based gain control: Divisive normalization in parietal cortex. J Neurosci, 31(29): 10627-10639.

Louie K., LoFaro T., Webb R., Glimcher P.W. (2014) Dynamic divisive normalization predicts time-varying value coding in decision-related circuits. J Neurosci. 34: 16046-16057.

Louie, K. L., and Glimcher, P. W. (2010). Separating value from choice: delay discounting activity in area LIP. J Neurosci. 30: 5498-5507.

MacLean, P. (1973) The triune concept of brain and behavior. Toronto: Univ of Toronto Press.

Mazur, JE. (1984) Tests of an equivalence rule for fixed and variable reinforcer delays. Journal of Experimental Psychology: Animal Behavior Processes. 10: 426-436.

Mazurek, ME and Shadlen, MN (2002) Limits to the temporal fidelity of cortical spike rate signals. Nature Neuroscience. 5: 463-471.

McClure, SM, Laibson, DI, Loewenstein, G and Cohen, JD. (2004) Separate neural systems value immediate and delayed monetary rewards. Science. 306: 503-507.

McFadden, D. (1974). Conditional logit analysis of qualitative choice behavior. In: P. Zarembka (ed.), Frontier in Econometrics. New York: Academic Press, pp. 105-142.

Mill, J.S. (1863) Utilitarianism. Parker, Son and Bourne: London.

Myerson, J. and Green, L. (1995) Discounting of delayed rewards: Models of individual choice. J Exp Anal Behav. 64: 263-276.

Padoa-Schioppa, C. (2009) Range-adapting representation of economic value in the orbitofrontal cortex. J Neurosci. 29: 14004-14014.

Pascal, B. (1623-1662; 1948). Great Shorter Works of Pascal, E. Cailliet and J. C. Blankenagel (trans.). Philadelphia: Westminster Press.

Plato. Rowe C (trans: 2005) Phaedrus. New York: Penguin.

Platt, M. L., and Glimcher, P. W. (1999). Neural correlates of decision variables in parietal cortex. Nature, 400: 233-238.

Polania R., Woodford M., and Ruff C. (2019) Efficient coding of subjective value. Nature Neuroscience. 22: 134-142.

Raichle, ME and Gusnard, DA. (2002) Appraising the brain's energy budget. PNAS. 99: 1023710239. 
Rawnsley, JH and Leeson, NW (1995) Total Risk: Nick Leeson and the Fall of Barings Bank. New York: Harper Collins.

Reynolds, J.H. and Heeger, D.J. (2009) The normalization model of attention. Neuron. 61: 168185.

Samuelson, P. A. (1937). A note on measurement of utility. Review of Economic Studies, 4: 155161.

Sato, TK, Haider, B, Häusser, M and Carandini, M. (2016) An excitatory basis for divisive normalization in visual cortex. Nature Neuroscience. 19: 568-570.

Savage, L. J. (1954). Foundations of Statistics. New York: John Wiley \& Sons, Inc.

Schwartz, O., and Simoncelli, E. P. (2001). Natural signal statistics and sensory gain control. Nature Neuroscience, 4(8): 819-825.

Shadlen, MN and Newsome, WT (1994) Noise, neural codes and cortical organization. Current Opinion in Neurobiology. 4: 569-579.

Shafir, S. (1994) Intransitivity of preferences in honey bees: support for 'comparative evaluation' of foraging options. Animal Behaviour. 48: 55-67.

Shannon, C.E. and Weaver, W. (1949) The mathematical theory of communication. Urbana: University of Illinois Press.

Simon, H. A. (1955). A behavioral model of rational choice. Quarterly Journal of Economics, 69(1): 99-118.

Simon, H. A. (1957). Models of Man: Social and Rational. New York: Wiley.

Simoncelli, E. P., and Heeger, D. J. (1998). A model of neuronal responses in visual area MT. Vision Research, 38: 743-761.

Sims, CA. (2003) Implications of rational inattention. Journal of Monetary Economics. 50: 665690.

Steverson, K, Brandenberger, A. and Glimcher PW (2019) Choice theoretic foundations of the divisive normalization model. JEBO. 164: 148-165.

Strotz, RH (1955) Myopia and inconsistency in dynamic utility maximization. REStud 23: 165180. 
Sugrue, L. P., Corrado, G. S., and Newsome, W. T. (2004). Matching behavior and the representation of value in the parietal cortex. Science, 304: 1782-1787.

Tajima, S, Drugowitsch, J, Patel, N and Pouget, A. (2019) Optimal policy for multi-alternative decisions. Nature neuroscience 22: 1503-1511.

Thaler, R. H. and Sunstein, C.R. (2003) Libertarian Paternalism. American Economic Review. 93: 175-179.

Tobler, P. N., Fiorillo, C. D., and Schultz, W. (2005). Adaptive coding of reward value by dopamine neurons. Science, 307: 1642-1645.

Tom, S. M., Fox, C. R., Trepel, C., and Poldrack, R. A. (2007). The neural basis of loss aversion in decision making under risk. Science, 315(5811): 515-518.

Tusche, A., Bode, S. \& Haynes, J.-D. (2010). Neural responses to unattended products predict later consumer choices. The Journal of Neuroscience, 30(23): 8024-8031.

Tversky, A., and Kahneman, D. (1986). Rational choice and the framing of decisions. Journal of Business 59: 251-278.

von Neumann, J. V., and Morgenstern, O. (1944). Theory of Games and Economic Behavior. Princeton, NJ: Princeton University Press.

Webb, R, Glimcher, PW and Louie, K. (2020) Divisive normalization does influence decisions with multiple alternatives. Nature Human Behavior. 4: 1118-1120.

Webb, R., Glimcher, PW, and Louie, K. (2020) The normalization of consumer valuations: Context-dependent preferences from neurobiological constraints. Management Science. 67: 93-125.

Wu, G., and Gonzalez, R. (1998). Common consequence effects in decision making under risk. Journal of Risk and Uncertainty, 16: 115-139.

Zimmermann, J., Glimcher, P.W. \& Louie, K. (2018) Multiple timescales of normalized value coding underlie adaptive choice behavior. Nat Commun 9, 3206. 